\title{
Actual Survival in Patients with Resected Pancreatic Cancer: How Do Real-World Data Compare with Clinical Trial Evidence?
}

\author{
Rebecca A. Snyder, MD, MPH, FSSO, and Alexander A. Parikh, MD, MPH, FSSO \\ Division of Surgical Oncology, Department of Surgery, Brody School of Medicine at East Carolina University, Greenville, \\ $\mathrm{NC}$
}

Within the last decade, the multimodality management of pancreatic cancer has evolved significantly, including changes in chemotherapeutic regimens as well as treatment sequencing. Encouragingly, the 5-year overall survival (OS) rate for all patients with pancreatic cancer recently reached $10 \%$ for the first time. ${ }^{1}$ Following the original GITSG trial published in 1985 and the ESPAC-1 trial published in 2001, single-agent 5-fluorouracil-based adjuvant chemotherapy became the mainstay of therapy in patients with resected pancreatic cancer, resulting in a median OS of approximately 20 months. ${ }^{2,3}$ More recently, the median OS in resected patients treated on clinical trial with newer adjuvant chemotherapy regimens increased from 23 months in the CONKO-001 trial (2007) to an unprecedented 54 months in the PRODIGE 24-ACCORD trial (2018). ${ }^{4,5}$ Although these results are promising to both patients and treating physicians, critics often question the 'real-world' generalizability of randomized controlled trials. On-treatment monitoring and compliance is enhanced for patients treated within a clinical trial, and the stringent inclusion criteria may select for patients with more favorable biology and a better performance status than the pancreatic cancer population at large.

The article by Belfiori et al. in this edition of Annals of Surgical Oncology provides a retrospective review of prospectively collected data in a cohort of 'real-world' patients with pancreatic cancer who underwent resection followed by adjuvant chemotherapy. ${ }^{6}$ The primary study

(C) Society of Surgical Oncology 2021

First Received: 7 July 2021

Accepted: 18 July 2021;

Published Online: 12 September 2021

A. A. Parikh, MD, MPH, FSSO

e-mail: parikha19@ecu.edu aim was to report actual survival, specifically 5-year disease-specific survival (DSS) and post-recurrence survival (PRS), to augment the survival estimates generated from randomized trials. At the time that this study was designed and conducted (2009-2014), standard of care consisted of adjuvant single-agent gemcitabine chemotherapy, based on the results of the CONKO-001 trial. ${ }^{3,7}$ Although singleagent gemcitabine has now been supplanted by multi-agent regimens, there is still clinical value in comparing these real-world results with those of the original CONKO-001 trial. $^{5}$

First, this study includes complete long-term follow-up, which allows determination of actual survival in a more heterogenous patient population treated off protocol, which is likely more reflective of clinical practice. Second, comparisons of these results with published trial data may allow us to better design future 'real-world' validation and applicability studies for the newer, more current regimens that are now standard of care. Third, the authors investigate not only DSS but also PRS, which, with the recent availability of multiple chemotherapeutic regimens, is an important and understudied outcome. Measurement of PRS informs our understanding of what happens once patients recur, which occurs in $80 \%$ of patients with pancreatic cancer following resection. ${ }^{8}$ Until recently, very few second-line agents have been effective in the treatment of recurrent disease, resulting in a short PRS. Indeed, advances in multi-agent therapeutic regimens may help explain the striking difference between the median diseasefree survival (DFS) and OS in both arms of the PRODIGE 24-ACCORD trial. ${ }^{4}$

CONKO-001, initially published in 2007 and updated in 2013, randomized 368 resected pancreatic cancer patients to 6 months of adjuvant gemcitabine or observation. ${ }^{5,9}$ At a median follow-up of 53 months, median DFS was 13.4 
months in the gemcitabine group compared with 6.9 months in the observation arm. Interestingly, median OS was not significantly different between groups (22.1 vs. 20.2 months). Publication of long-term results illustrated a median DFS of 13.4 months in the gemcitabine arm (vs. 6.7 months) and significantly improved OS (22.8 months vs. 20.2 months). In contrast to many clinical trials, CONKO001 was conducted at both academic and community centers without uniform standards for surgical resection or centralized pathologic review. Although this may question some of the reliability of staging and pathology data, CONKO-001 results may represent real-world evidence more generalizable to the general population than most multidisciplinary trials.

In comparison, Belfiori et al. reported a median DFS of 19 months, PRS of 12 months, and DSS of 36 months in a cohort of 176 patients followed for a median of 38 months. ${ }^{6}$ Of these, 166 (94\%) were treated with adjuvant gemcitabine, although no data are provided regarding treatment completion rates, delays, or dose adjustments. However, the study by Belfiori et al. addresses a critical gap in knowledge, as actual survival, not estimated survival, as well as survival following recurrence of disease, are not reported in either clinical trials or large, populationbased cohort studies. Unfortunately, however, applicability of these data to contemporary clinical practice is limited, as gemcitabine monotherapy is no longer standard nor commonly used in the adjuvant setting.

A key consideration in the interpretation of the accompanying study is the difference between actual survival and Kaplan-Meier estimates or actuarial survival as reported in clinical trials. Actual survival implies that complete followup data are available for all patients in the study cohort, and thereby eliminates assumptions made for censored patients in usual Kaplan-Meier estimates. Generally speaking, actual survival is more accurate, although Kaplan-Meier estimates are commonly used due to the limited feasibility of following every patient to study completion. In their cohort selection, the present study excluded five patients who were later lost to follow-up, thereby exposing their results to at least some potential selection bias.

In comparing the patient cohorts in the study by Belfiori et al. and the CONKO-001 trial, it is important to note that although the $\mathrm{T}$ and $\mathrm{N}$ stage were similar, the positive (R1) margin rate in CONKO-001 ranged between 15 and 19\%, whereas Belfiori et al. reported a rate of $42 \% .^{6,9}$ The reasons for this are unclear and could represent differences in surgical quality control, pathologic assessment, or, less likely, tumor biology. Interestingly, the R1 margin rate reported in the PRODIGE 24-ACCORD trial was $40-45 \%{ }^{4}$ This is important to consider when examining recurrence patterns, as those with positive margins experience increased risk of local recurrence. ${ }^{8}$
Finally, in the study by Belfiore et al., the surveillance interval was short, consisting of carbohydrate antigen (CA) 19-9 and computed tomography (CT) imaging every 3-4 months for the first 2 years and every 6 months in years 3-5. Data regarding asymptomatic versus symptomatic recurrence is not provided. Although this schedule is a commonly utilized surveillance strategy, there is no highlevel evidence that more frequent surveillance testing is associated with an increased likelihood of curative treatment of recurrent disease or with OS in patients with pancreatic cancer. In the context of this frequent testing schedule, the authors report a median time to recurrence of 19 months, which calls into question the necessity of frequent asymptomatic testing. Even in patients with colorectal cancer with a multitude of local and systemic treatment options, increased intensity of surveillance testing has not been demonstrated to yield improvements in OS. ${ }^{10-12}$ Therefore, less frequent, or even symptom-driven surveillance testing, should be considered, especially in the setting of aggressive disease biology, such as pancreatic cancer. Surprisingly, in this study, $45 \%$ of patients with recurrent disease had isolated local recurrence, a proportion much higher than expected in what is typically considered a systemic disease. This may be secondary to the high margin positivity rate in this study.

The authors suggest that "effective treatment of recurrence is also of paramount importance to allow a favorable post-recurrence survival (PRS)", based on an odds ratio of 7.12 among patients treated for recurrence compared with those not treated. However, the observed PRS survival benefit is less likely to represent actual treatment efficacy, and more likely to represent selection bias of a specific subset of patients eligible for treatment, typically those with good functional status, limited comorbidities, isolated or limited disease recurrence, and more favorable biology. The regimens used in patients who did recur in this study were heterogeneous, including both monotherapy and multi-agent systemic chemotherapy as well as chemoradiation. In addition, it is not clear whether starting treatment for asymptomatic patients with recurrence confers any survival benefit compared with waiting until these patients become symptomatic.

How should we incorporate the findings of the current study into clinical practice? Whereas the purpose of randomized clinical trials is to test new therapeutic options compared with the existing standard of care, the value of studies such as the study by Belfiore et al. is the application and validation of new treatment standards to the general population of patients with pancreatic cancer outside of a clinical trial. Critics have suggested that the impressive median OS estimate of 54 months in resected patients treated with adjuvant FOLFIRINOX on the PRODIGE 24-ACCORD trial represents a highly preselected cohort of 
patients already likely to experience a favorable prognosis, i.e. patients healthy enough to successfully undergo pancreatectomy without major morbidity. Whether this treatment approach yields equivalent actual survival rates in a real-world setting is yet to be seen, and future studies similar to the study by Belfiori et al. may help to answer this question.

\section{REFERENCES}

1. SEER Cancer Stat Facts: Pancreatic Cancer. National Cancer Institute. Available at: https://seer.cancer.gov/statfacts/html/panc reas.html. Accessed 5 Jul 2021.

2. Kalser MH, Ellenberg SS. Pancreatic cancer. Adjuvant combined radiation and chemotherapy following curative resection. Arch Surg. 1985;120(8):899-903.

3. Neoptolemos JP, Dunn JA, Stocken DD, et al. Adjuvant chemoradiotherapy and chemotherapy in resectable pancreatic cancer: a randomised controlled trial. Lancet. 2001;358(9293):1576-85.

4. Conroy T, Hammel P, Hebbar M, et al. FOLFIRINOX or gemcitabine as adjuvant therapy for pancreatic cancer. $N$ Engl $J$ Med. 2018;379(25):2395-406.

5. Oettle H, Post S, Neuhaus P, et al. Adjuvant chemotherapy with gemcitabine vs observation in patients undergoing curative-intent resection of pancreatic cancer: a randomized controlled trial. JAMA. 2007;297(3):267-77.

6. Belfiori G, Crippa S, Francesca A, Pagnanelli M, Tamburrino D, Gasparini G, et al. Long-term survivors after upfront resection for pancreatic ductal adenocarcinoma: an actual 5-year analysis of disease specific and post-recurrence survival. Ann Surg Oncol. 2021. https://doi.org/10.1245/s10434-021-10401-7.

7. Neoptolemos JP, Stocken DD, Friess H, et al. A randomized trial of chemoradiotherapy and chemotherapy after resection of pancreatic cancer. N Engl J Med. 2004;350(12):1200-10.

8. Tanaka M, Mihaljevic AL, Probst P, et al. Meta-analysis of recurrence pattern after resection for pancreatic cancer. Br J Surg. 2019;106(12):1590-601.

9. Oettle H, Neuhaus P, Hochhaus A, et al. Adjuvant chemotherapy with gemcitabine and long-term outcomes among patients with resected pancreatic cancer: the CONKO-001 randomized trial. JAMA. 2013;310(14):1473-81.

10. Primrose JN, Perera R, Gray A, et al. Effect of 3 to 5 years of scheduled CEA and CT follow-up to detect recurrence of colorectal cancer: the FACS randomized clinical trial. JAMA. 2014;311(3):263-70.

11. Snyder RA, Hu CY, Cuddy A, et al. Association between intensity of posttreatment surveillance testing and detection of recurrence in patients with colorectal cancer. JAMA. 2018;319(20):2104-15.

12. Wille-Jørgensen P, Syk I, Smedh K, et al. Effect of more vs less frequent follow-up testing on overall and colorectal cancerspecific mortality in patients with stage II or III colorectal cancer: the COLOFOL randomized clinical trial. JAMA. 2018;319(20):2095-103.

Publisher's Note Springer Nature remains neutral with regard to jurisdictional claims in published maps and institutional affiliations. 